\title{
MODELO DE REGRESIÓN DEL CONSUMO FINAL PRIVADO Y EL PRODUCTO BRUTO INTERNO NACIONAL DEL PERÚ DURANTE EL PERIODO 2000 - 2013
}

\author{
MODEL REGRESSION OF PRIVATE FINAL CONSUMPTION AND \\ THE NATIONAL INTERNAL GROSS PRODUCT OF PERU \\ DURING THE PERIOD 2000 - 2013
}

Carmen Rosa Barreto Rodríguez ${ }^{1}$

\section{RESUMEN}

Esta investigación tuvo como propósito estimar un modelo de regresión para el Consumo Final Privado sobre el Producto Bruto Interno Nacional (PBI) del Perú en el periodo 2000 2013 utilizando el método de los mínimos cuadrados ordinarios (MCO) con la finalidad de realizar predicciones. Se consideró a la variable Consumo Final Privado como variable dependiente y al Producto Bruto Interno Nacional (PBI) como variable independiente las cuales están expresadas en millones de soles a valores de precios constantes del 2007. Para determinar el modelo de regresión estimado se utilizó el software estadístico SPSS, versión 22, el cual permitió hacer un análisis previo de los modelos de regresión reducible a lineal de tipo exponencial, potencial, logarítmico e inverso y otros, mediante los gráficos de dispersión, error estándar de estimación, ANOVA y coeficientes de regresión estimados. Los resultados nos indican que el modelo de regresión estimado es un modelo exponencial, el cual nos indica que el Consumo Final Privado depende exponencialmente del Producto Bruto Interno Nacional (PBI) mediante el cual se hicieron predicciones para los años 2014, 2015 y 2016, en base a la información brindada por el INEI.

PALABRAS CLAVE: Bondad de ajuste, consumo final privado, PBI, modelo de regresión.

1 Magíster en Ciencias de la Educación con mención en Docencia Universitaria por la Universidad Nacional Pedro Ruiz Gallo. Docente asociada de la Escuela de Ingeniería de Sistemas .Universidad Católica Los Ángeles de Chimbote. 


\begin{abstract}
The purpose of this research was to estimate a regression model for Private Final Consumption on the National Gross Domestic Product (GDP) of Peru in the period 2000 - 2013 using the ordinary least squares (OLS) method in order to make predictions. The Final Private Consumption variable was considered as a dependent variable and the National Gross Domestic Product (GDP) as an independent variable, which are expressed in millions of soles at constant price values of 2007. To determine the estimated regression model, the software was used statistical SPSS, version 22, which allowed to make a previous analysis of the models of reducible to linear regression of exponential, potential, logarithmic and inverse type, etc. by means of dispersion graphs, standard estimation error, ANOVA and estimated regression coefficients. The results indicate that the estimated regression model is an exponential model, which indicates that Private Final Consumption depends exponentially on the National Gross Domestic Product (GDP) through which predictions were made for the years 2014, 2015 and 2016, in based on the information provided by the INEI.
\end{abstract}

KEY WORDS: Private final consumption, GDP, regression model, goodness of fit.

\title{
INTRODUCCIÓN
}

El consumo privado es una variable especialmente importante en el estudio macroeconómico y mide el valor total en un periodo de los bienes y servicios que los hogares, empresas o instituciones privadas adquieren dentro de sus correspondientes actividades económicas. Al mismo tiempo, la propia definición de consumo establece que este gasto es realizado en bienes finales y no en otros dirigidos a la producción de otros.

El Producto Bruto Interno Nacional o Renta Nacional de un país consiste, básicamente, en la suma del consumo privado, la inversión privada, el gasto público y el saldo exterior neto. Como el consumo privado es la parte proporcional más importante de dicho sumando, las variaciones del consumo tienen consecuencias a corto plazo muy importantes en las variaciones de la producción, de la renta, del empleo y, por tanto, son determinantes fundamentales de los ciclos económicos.

Según Instituto Nacional de Estadística (2015) expresa que, en el tercer trimestre de 2015, el consumo final privado creció 3,3\% reflejado en el mayor gasto de los hogares como resultado del aumento en el ingreso promedio por trabajo en 4,4\% y el número de personas ocupadas en el país que crecieron en 1,7\%. También manifiesta que en dicho período se incrementó el otorgamiento de los créditos de consumo (46,9\%), reportados por la Superintendencia Nacional de Banca y Seguros, y que este comportamiento positivo coadyuvó al crecimiento de 
la actividad económica, medida a través del Producto Bruto Interno (PBI), a precios constantes de 2007, que aumentó en 2.9\% en dicho trimestre.

El INEI (2016) revela que, en el segundo trimestre del año 2016, el Producto Bruto Interno (PBI) se incrementó en 3,7\% influenciado por el mayor gasto de consumo final público y privado; así como el aumento de las exportaciones de bienes y servicios.

Según el INEI (2017) afirma que el gasto de consumo final privado creció en 2,3\% en el tercer trimestre del año 2017 respecto a similar periodo del año anterior. En términos nominales el gasto de consumo final privado representó el $64,2 \%$ del PBI.

En un estudio realizado Rodríguez (2006) en su artículo publicado Análisis de Regresión Simple de Consumo Privado en América Latina (Colombia y Bolivia) expresa que respecto a Colombia los residuos no tienen un alto grado de variación, por lo mismo el coeficiente de correlación de datos los estima en un 99\% aproximadamente y que por cada millón de dólares que suba el PIB, el consumo privado aumenta en unos 0,6285 millones de dólares. Respecto a Bolivia expresa que por cada millón de dólares que aumente el PIB, el consumo privado aumenta en 0,6533 millones de dólares, el coeficiente correlación evidencia un 99\% de exactitud, a diferencia de que en Colombia dicho coeficiente de correlación es mucho más exacto.

Según Marulanda (2016) en un post sobre un análisis del PIB per cápita y del consumo privado en la economía española, con los datos del Banco Mundial, desde 1970 hasta 2014 afirma que "se puede decir que el ciclo del consumo privado es una variable fuertemente procíclica del PIB per cápita al presentar un coeficiente de correlación del $92 \%$.

Como podemos ver en ambos países Colombia y Bolivia el Consumo Privado están expresados linealmente por el Producto Bruto Interno.

Dado que el consumo privado final tiene una mayor participación en el PBI El interés de esta investigación radica en determinar un modelo de regresión que permita representar estas variables. El Consumo Final Privado como variable dependiente y el PBI como variable independiente.

Ante esto se planteó el siguiente problema ¿Qué modelo de regresión representa adecuadamente al Consumo Final Privado en función del Producto Bruto Interno Nacional? 
El objetivo general es estimar el modelo de regresión del Consumo Final Privado sobre el Producto Bruto Interno Nacional.

Los objetivos específicos de esta investigación fueron: analizar el modelo de regresión del Consumo Final Privado sobre el Producto Bruto Interno Nacional y pronosticar el comportamiento del Consumo Final Privado en función del Producto Bruto Interno Nacional para los años 2014, 2015 y 2016.

La importancia de esta investigación radica en conocer qué modelo de regresión se ajusta a conjunto de datos en estudio con un menor error posible para realizar predicciones y sirva de apoyo para otros estudios similares.

Esta investigación tiene sus bases teóricas en los modelos de regresión reducible a lineal entre los más usados de tipo exponencial, potencial, logarítmica e inversa mediante el método de los mínimos cuadrados ordinarios (MCO).

Donde «y» es la variable dependiente $\mathrm{y}$ « $\mathrm{x}$ l la variable independiente.

\section{Modelo de regresión exponencial se expresa como: $\boldsymbol{y}=\boldsymbol{a} \cdot \boldsymbol{b}^{\boldsymbol{x}}$}

Tomandologaritmos en la $y=a \cdot b^{x}$ se tiene:

$$
\ln y=\ln a+x \cdot \ln b
$$

Designando: $z=\ln y ; A=\ln a y B=\ln b$

Entonces se tiene: $z=A+B x$ para lo cual se utiliza el método de los mínimos cuadrados para estimar $A$ y $B$.

El método de los mínimos cuadrados ordinarios (MCO) se utiliza para determinar los coeficientes de regresión estimados, en este caso A y B.

Donde:

$$
\begin{aligned}
& B=\frac{n \sum_{i=1}^{n} x_{i} z_{i}-\left(\sum_{i=1}^{n} x_{i}\right) \cdot\left(\sum_{i=1}^{n} z_{i}\right)}{n \sum_{i=1}^{n} x_{i}^{2}-\left(\sum_{i=1}^{n} x_{i}\right)^{2}} \\
& A=\bar{z}-B \bar{x}
\end{aligned}
$$

Un caso particular de regresión exponencial es el de la ecuación $y=a \cdot e^{b x}$ que es el que utilizaremos para representar los datos en estudio.

Tomando logaritmos obtenemos: $\ln y=\ln a+m x$

Designando $a: z=\ln y ; A=\ln a$ se tiene $y=A+m x$. 


\section{Modelo de regresión potencial se expresa como: $\boldsymbol{y}=\boldsymbol{a} \cdot \boldsymbol{x}^{\boldsymbol{b}}$}

Tomando logaritmos en la $y=a \cdot x^{b}$ se tiene:

$$
\ln y=\ln a+b \cdot \ln x
$$

Designando: $z=\ln y ; A=\ln a y u=\ln x$

Entonces se tiene: $z=A+b u$ para lo cual se utiliza el método de los mínimos cuadrados para estimar A yb.

El método de los mínimos cuadrados ordinarios (MCO) se utiliza para determinar los coeficientes de regresión estimados, en este caso A y b.

Donde:

$$
\begin{aligned}
b & =\frac{n \sum_{i=1}^{n} u_{i} z_{i}-\left(\sum_{i=1}^{n} u_{i}\right) \cdot\left(\sum_{i=1}^{n} z_{i}\right)}{n \sum_{i=1}^{n} u_{i}^{2}-\left(\sum_{i=1}^{n} u_{i}\right)^{2}} \\
A & =\bar{z}-b \bar{u}
\end{aligned}
$$

Modelo de regresión logarítmico se expresa como: $\boldsymbol{y}=\boldsymbol{a}+\boldsymbol{b} \ln x$

Designando: $u=\ln x$

Entonces se tiene: $y=a+b u$ para lo cual se utiliza el método de los mínimos cuadrados para estimar a y b.

El método de los mínimos cuadrados ordinarios (MCO) se utiliza para determinar los coeficientes de regresión estimados, en este caso a y b.

Donde:

$$
\begin{aligned}
& b=\frac{n \sum_{i=1}^{n} u_{i} y_{i}-\left(\sum_{i=1}^{n} u_{i}\right) \cdot\left(\sum_{i=1}^{n} y_{i}\right)}{n \sum_{i=1}^{n} u_{i}^{2}-\left(\sum_{i=1}^{n} u_{i}\right)^{2}} \\
& A=\bar{y}-b \bar{u}
\end{aligned}
$$

Modelo de regresión inverso se expresa como: $\boldsymbol{y}=\boldsymbol{a}+\frac{\boldsymbol{b}}{\boldsymbol{x}}$

Designando: $z=\frac{1}{x}$

Entonces se tiene: $y=a+z b$ para lo cual se utiliza el método de los mínimos cuadrados para estimar a y $b$.

El método de los mínimos cuadrados ordinarios (MCO) se utiliza para determinar los coeficientes de regresión estimados, en este caso a y b. 
Donde:

$$
\begin{aligned}
& b=\frac{n \sum_{i=1}^{n} z_{i} y_{i}-\left(\sum_{i=1}^{n} z\right) \cdot\left(\sum_{i=1}^{n} y_{i}\right)}{n \sum_{i=1}^{n} z-\left(\sum_{i=1}^{n} z_{i}\right)^{2}} \\
& a=\bar{y}-b \bar{z}
\end{aligned}
$$

Según Webster (2001) expresa que hay por los menos dos medidas de bondad de ajuste: el error estándar de estimación (Se) y el coeficiente de determinación (r2). El error estándar de estimación mide la variación de los puntos por encima y por debajo de la recta de regresión. El coeficiente de determinación determina la calidad del modelo para replicar los resultados, y la proporción de variación de los resultados que puede explicarse por el modelo.

De ser "y" la variable independiente y "x" la variable dependiente

El error estándar de estimación se expresa como:

$$
S e=\sqrt{\frac{\sum_{i=1}^{n}\left(y_{i}-\hat{y}_{i}\right)^{2}}{n-2}}
$$

De otro modo, Pedraza (2014) afirma que el error estándar de estimación trata de resumir la disparidad entre lo estimado y observado, es decir tratar de medir la diferencia promedio entre lo observado y estimado o esperado de acuerdo con el modelo, puede considerarse como un indicador del grado de precisión con que la ecuación de regresión describe la relación entre las dos variables.

El coeficiente de determinación está dado por:

$$
r^{2}=\frac{n \sum_{i=1}^{n} x_{i} y_{i}-\left(\sum_{i=1}^{n} x_{i}\right) \cdot\left(\sum_{i=1}^{n} y_{i}\right)}{\sqrt{\left(n \sum_{i=1}^{n} x_{i}^{2}-\left(\sum_{i=1}^{n} x_{i}\right)^{2}\right) \cdot\left(n \sum_{i=1}^{n} y_{i}^{2}-\left(\sum_{i=1}^{n} y_{i}\right)^{2}\right)}}
$$

Según Garcia, Gonzales \& Ruiz (2016) expresa que el coeficiente de determinación $r^{2}$ tiene un rango de variación acotado $\left(0 \leq r^{2} \leq 1\right)$. $\operatorname{Si~}^{2}=1$ quiere decir que el ajuste es perfecto. $\mathrm{Si} \mathrm{r}^{2}=0$ no se consigue ninguna explicación de la variable "y" relacionada con la variable "x" mediante la curva considerada. $\mathrm{Si}^{2}=0,75$, el modelo ajustado suele aceptarse, si el coeficiente es menor a dicho valor, concluiremos que la relación elegida no es adecuada, debiendo ensayar con otro tipo de función. 


\section{MATERIAL Y MÉTODOS}

La investigación realizada sigue un diseño no experimental de tipo longitudinal de tendencia. Se realiza esta investigación pues no se realiza una manipulación deliberada de la variable de interés, es decir, se trata de un estudio donde no hacemos variar en forma intencional la variable independiente. Lo que se realiza en la investigación no experimental es observar fenómenos tal como se da en su contexto natural para después analizarlos (Hernández, R., Fernández, C., \& Baptista, P., 2006).

La población está constituida por las series temporales del Consumo Final Privado y el PBI del Perú desde 1950 al 2016 en millones de soles a precios constantes del 2007.

Para la selección de la muestra se ha tomado en cuenta lo que indica De la Fuente, Pino \& Parreño (1995):

Aunque en una serie temporal se dispone de una observación por cada periodo de tiempo, esta no se obtiene generalmente de una manera determinista, sino que tiene un carácter aleatorio. Se puede interpretar una serie temporal como una muestra de tamaño 1 , tomada en periodos sucesivos de tiempo en un proceso estocástico. Se considera por lo tanto a una serie temporal como una realización de un proceso estocástico. A diferencia del muestreo aleatoria simple, donde cada extracción es independiente de las demás, en una serie temporal el dato extraído para un periodo concreto no va ser independiente en general, de los extraídos en periodos anteriores.

La muestra aleatoria a considerar en esta investigación está dada por las series temporales del Consumo Final Privado y el PBI del Perú desde 2000 al 2013 en millones de soles a precios constantes del 2007, lo cual constituye una muestra de $n=14$ observaciones.

\section{RESULTADOS}

El Software SPSS versión 22 brinda los siguientes resultados respecto a los coeficientes de regresión estimados, coeficiente de determinación, error típico(estándar) de estimación, ANOVA, así como los gráficos respectivos del diagrama de dispersión y curvas de regresión estimadas. 
Carmen Rosa Barreto Rodríguez

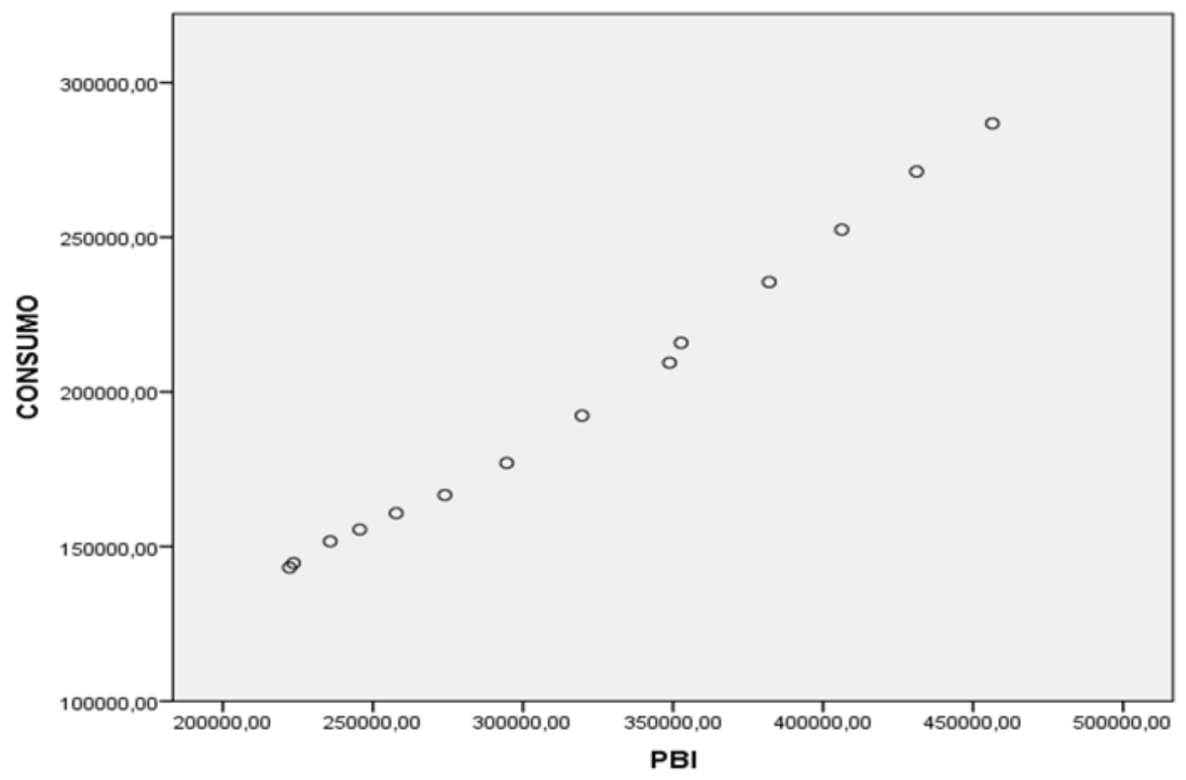

Fuente: Elaboración propia.

Gráfico 1. Diagrama de dispersión del Consumo Final Privado sobre el PBI.

Cuadro 1

COEFICIENTE DE REGRESIÓN ESTIMADOS DEL MODELO EXPONENCIAL ESTIMADO

\begin{tabular}{|c|c|c|c|c|c|}
\hline & \multicolumn{2}{|c|}{ Coeficientes no estandarizados } & $\begin{array}{c}\text { Coeficientes } \\
\text { estandarizados }\end{array}$ & \multirow{2}{*}{$t$} & \multirow{2}{*}{ Sig. } \\
\cline { 2 - 5 } & B & Error estándar & Beta & & \\
\hline PBI & $3,003 \mathrm{E}-006$ & 0,000 & 0,999 & 90,707 & 0,000 \\
\hline (Constante) & 73936,560 & 800,544 & & 92,358 & 0,000 \\
\hline
\end{tabular}

La variable dependiente es $\ln (\mathrm{CONSUMO})$. 
Contraste individual de los coeficientes de regresión:

Para b:

Ho: $b=0$

$\mathrm{H} 1: \mathrm{b} \neq 0$

El valor $\mathrm{t}=90,707$ tiene un valor $\mathrm{p}$-valor $=0,000<0,05$ por lo que rechazamos la hipótesis nula, lo cual nos indica que $(b \neq 0)$.

\section{Para a:}

Ho: $\mathrm{a}=0$

$\mathrm{H} 1: \mathrm{a} \neq 0$

El valor $\mathrm{t}=92,358$ tiene un valor $\mathrm{p}$-valor $=0,000<0,05$ por lo que rechazamos la hipótesis nula, lo cual indica que $(a \neq 0)$.

Cuadro 2

COEFICIENTE DE DETERMINACIÓN Y ERROR TÍPICO DE ESTIMACIÓN DEL MODELO DE REGRESIÓN EXPONENCIAL ESTIMADO

\begin{tabular}{|c|c|c|}
\hline$r$ & $r^{2}$ & Error estándar de la estimación \\
\hline 0,999 & 0,999 & 0,009 \\
\hline
\end{tabular}

Cuadro 3

ANOVA DEL MODELO DE REGRESIÓN EXPONENCIAL ESTIMADO

\begin{tabular}{|l|c|c|c|c|c|}
\hline & $\begin{array}{c}\text { Sumade } \\
\text { cuadrados }\end{array}$ & $g l$ & $\begin{array}{c}\text { Media } \\
\text { cuadrática }\end{array}$ & F & Sig. \\
\hline Regresión & 0,742 & 1 & 0,742 & 8227,744 & 0,000 \\
\hline Residual & 0,001 & 12 & 0,000 & & \\
\hline Total & 0,743 & 13 & & & \\
\hline
\end{tabular}

La variable independiente es PBI. 


\section{Contraste de regresión:}

Ho: $b=0$ (El modelo de regresión exponencial no es significativo).

$\mathrm{H} 1: \mathrm{b} \neq 0$ (El modelo de regresión exponencial es significativo).

$\mathrm{El}$ valor $\mathrm{F}=8227,744$ tiene un valor $\mathrm{p}$-valor $=0,000<0,05$ por lo que rechazamos la hipótesis nula, entonces $b \neq 0$; lo cual nos indica que el modelo de regresión exponencial es significativo.

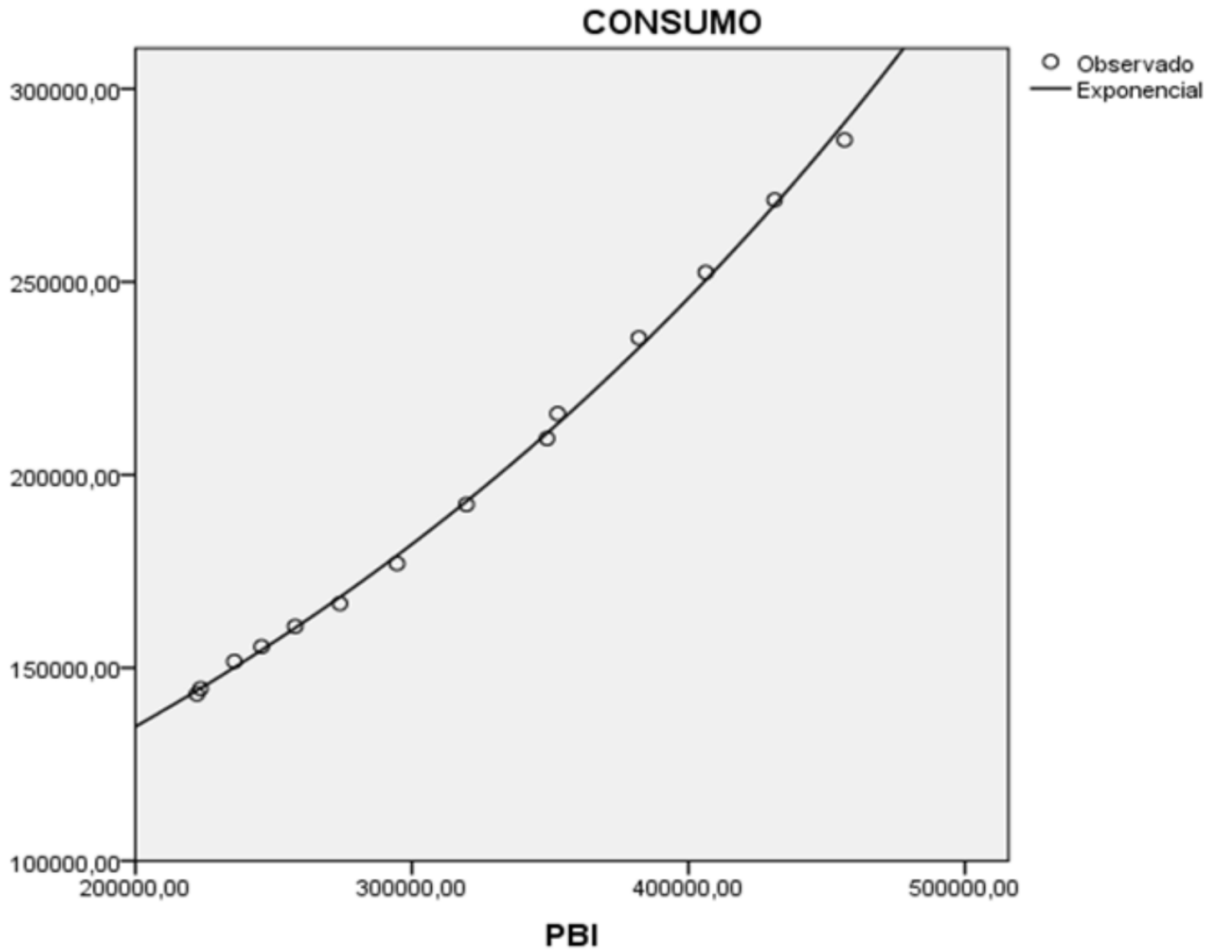

Gráfico 2. Curva de regresión exponencial estimada.

Modelo de regresión exponencial estimado:

$$
y=73936,560 e^{3,003 * 10^{-6} x}
$$

Resultados del modelo de regresión potencial: 


\section{Cuadro 4 \\ COEFICIENTE DE REGRESIÓN ESTIMADOS DEL MODELO POTENCIAL ESTIMADO}

\begin{tabular}{|c|c|c|c|c|c|}
\hline & \multicolumn{2}{|c|}{ Coeficientes no estandarizados } & $\begin{array}{c}\text { Coeficientes } \\
\text { estandarizados }\end{array}$ & \multirow{2}{*}{$t$} & \multirow{2}{*}{ Sig. } \\
\cline { 2 - 5 } & $B$ & Error estándar & Beta & & \\
\hline In(PBI) & 0,956 & 0,027 & 0,995 & 34,767 & 0,000 \\
\hline (Constante) & 1,090 & 0,379 & & 2,878 & 0,014 \\
\hline
\end{tabular}

La variable dependiente es $\ln (\mathrm{CONSUMO})$.

Contraste individual de los coeficientes de regresión:

\section{Para b:}

Ho: $b=0$

$\mathrm{H} 1: \mathrm{b} \neq 0$

El valor $\mathrm{t}=34,767$ tiene un valor $\mathrm{p}$-valor $=0,000<0,05$ por lo que rechazamos la hipótesis nula, lo cual nos indica que $(b \neq 0)$.

\section{Para a:}

Ho: $a=0$

$\mathrm{H} 1: \mathrm{a} \neq 0$

El valor $\mathrm{t}=2,878$ tiene un valor $\mathrm{p}$-valor $=0,000<0,05$ por lo que rechazamos la hipótesis nula, lo cual indica que $(a \neq 0)$.

\section{Cuadro 5}

COEFICIENTE DE DETERMINACIÓN Y ERROR ESTÁNDAR DE ESTIMACIÓN DEL MODELO DE REGRESIÓN POTENCIAL ESTIMADO

\begin{tabular}{|c|c|c|}
\hline$R$ & $R$ cuadrado & Error estándar de la estimación \\
\hline 0,995 & 0,990 & 0,025 \\
\hline
\end{tabular}


Cuadro 6

ANOVA DEL MODELO DE REGRESIÓN POTENCIAL ESTIMADO

\begin{tabular}{|l|c|c|c|c|c|}
\hline & $\begin{array}{c}\text { Sumade } \\
\text { cuadrados }\end{array}$ & $g l$ & $\begin{array}{c}\text { Media } \\
\text { cuadrática }\end{array}$ & F & Sig. \\
\hline Regresión & 0,736 & 1 & 0,736 & 1208,770 & 0,000 \\
\hline Residual & 0,007 & 12 & 0,001 & & \\
\hline Total & 0,743 & 13 & & & \\
\hline
\end{tabular}

La variable independiente es PBI.

\section{Contraste de regresión:}

Ho: $b=0$ (El modelo de regresión potencial no es significativo).

$\mathrm{H} 1: \mathrm{b} \neq 0$ (El modelo de regresión potencial es significativo).

El valor $\mathrm{F}=1208,770$ tiene un valor $\mathrm{p}$-valor $=0,000<0,05$ por lo que rechazamos la hipótesis nula, entonces $b \neq 0$; lo cual nos indica que el modelo de regresión potencial es significativo.

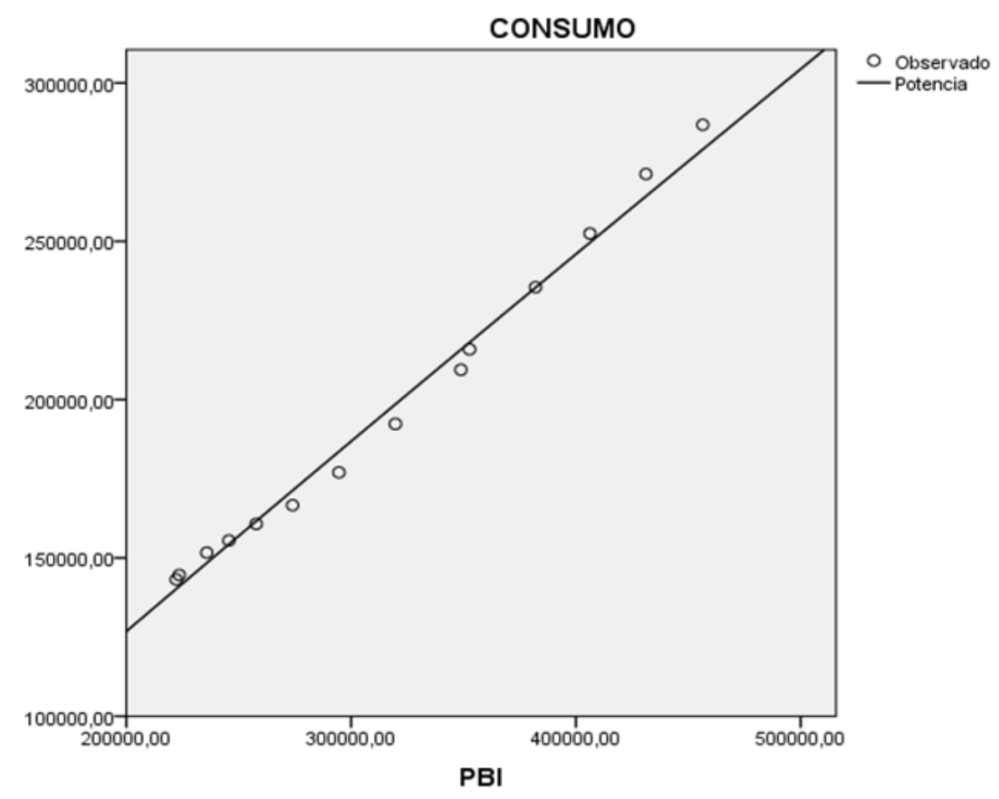

Gráfico 3. Curva del modelo de regresión potencial estimado. 
Resultados del modelo de regresión logarítmico:

Cuadro 7

COEFICIENTE DE REGRESIÓN ESTIMADOS DEL MODELO LOGARÍTMICO ESTIMADO

\begin{tabular}{|c|c|c|c|c|c|}
\hline & \multicolumn{2}{|c|}{ Coeficientes no estandarizados } & $\begin{array}{c}\text { Coeficientes } \\
\text { estandarizados }\end{array}$ & \multirow{2}{*}{$t$} & \multirow{2}{*}{ Sig. } \\
\cline { 2 - 5 } & $B$ & Error estándar & Beta & & \\
\hline In(PBI) & 191959,434 & 10333,114 & 0,983 & 18,577 & 0,000 \\
\hline (Constante) & $-2229138,657$ & 130641,084 & & $-17,063$ & 0,000 \\
\hline
\end{tabular}

Contraste individual de los coeficientes de regresión:

Para b:

Ho: $b=0$

$\mathrm{H} 1: \mathrm{b} \neq 0$

El valor $\mathrm{t}=18,577$ tiene un valor $\mathrm{p}$-valor $=0,000<0,05$ por lo que rechazamos la hipótesis nula, lo cual nos indica que $(b \neq 0)$.

\section{Para a:}

Ho: $\mathrm{a}=0$

$\mathrm{H} 1: \mathrm{a} \neq 0$

El valor $\mathrm{t}=-17,063$ tiene un valor $\mathrm{p}$-valor $=0,000<0,05$ por lo que rechazamos la hipótesis nula, lo cual indica que $(a \neq 0)$.

Cuadro 8

COEFICIENTE DE DETERMINACIÓN Y ERROR ESTÁNDAR DE ESTIMACIÓN DEL MODELO DE REGRESIÓN LOGARÍTMICO ESTIMADO

\begin{tabular}{|c|c|c|}
\hline$R$ & $R$ cuadrado & Error estándar de la estimación \\
\hline 0,983 & 0,966 & 9273,839 \\
\hline
\end{tabular}


Cuadro 9

ANOVA DEL MODELO DE REGRESIÓN LOGARÍTMICO ESTIMADO

\begin{tabular}{|l|c|c|c|c|c|}
\hline & Suma de cuadrados & $g l$ & Media cuadrática & F & Sig. \\
\hline Regresión & 29680795201,626 & 1 & 29680795201,626 & 345,109 & 0,000 \\
\hline Residual & 1032049033,231 & 12 & 86004086,103 & & \\
\hline Total & 30712844234,857 & 13 & & & \\
\hline
\end{tabular}

La variable independiente es PBI.

\section{Contraste de regresión:}

Ho: $b=0$ (El modelo de regresión logarítmico no es significativo).

$\mathrm{H} 1: \mathrm{b} \neq 0$ (El modelo de regresión logarítmico es significativo).

El valor $\mathrm{F}=345,109$ tiene un valor $\mathrm{p}$-valor $=0,000<0,05$ por lo que rechazamos la hipótesis nula, entonces $b \neq 0$; lo cual nos indica que el modelo de regresión logarítmico es significativo.

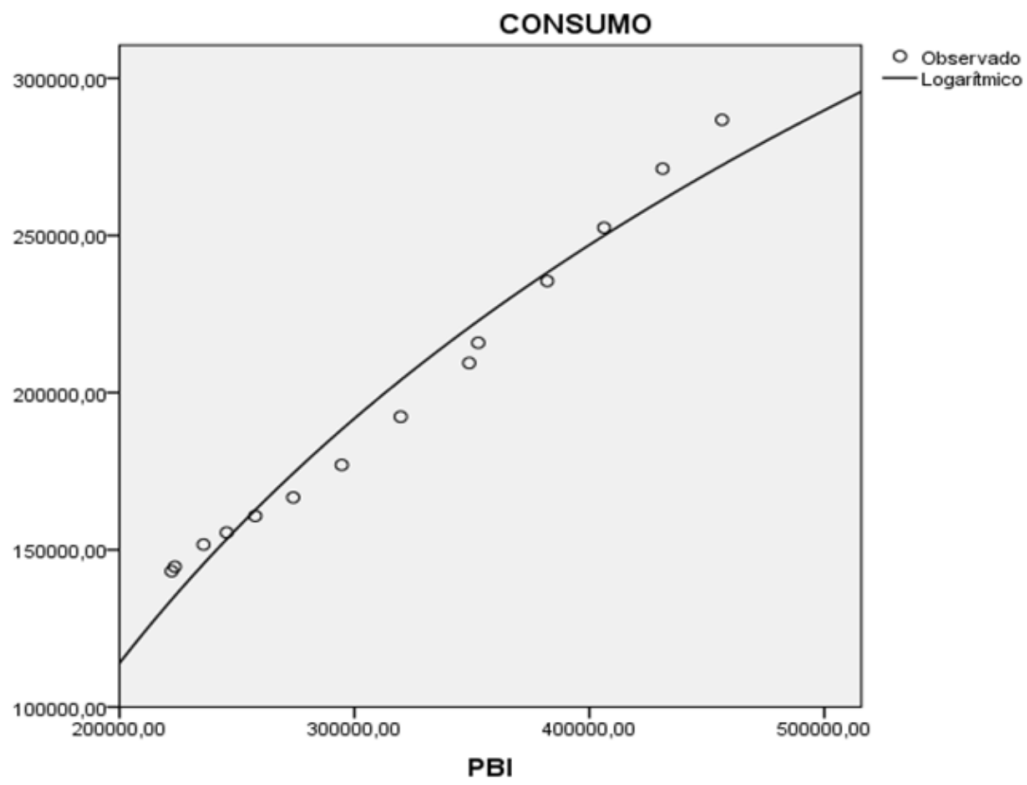

Gráfico 4. Curva del modelo de regresión logarítmico estimado. 
Modelo de regresión logarítmico estimado:

$y=-2229138,657+191959,934 \ln x$

Resultados del modelo de regresión inverso:

Cuadro 10

COEFICIENTE DE REGRESIÓN ESTIMADOS DEL MODELO INVERSO ESTIMADO

\begin{tabular}{|c|c|c|c|c|c|}
\hline & \multicolumn{2}{|c|}{ Coeficientes no estandarizados } & $\begin{array}{c}\text { Coeficientes } \\
\text { estandarizados }\end{array}$ & \multirow{2}{*}{$t$} & \multirow{2}{*}{ Sig. } \\
\cline { 2 - 5 } & B & Error típico & Beta & \\
\hline $1 /$ PBI & $-57673466510,027$ & 4719402030,451 & $-0,962$ & $-12,221$ & 0,000 \\
\hline (Constante) & 389446,419 & 16145,207 & & 24,121 & 0,000 \\
\hline
\end{tabular}

Contraste individual de los coeficientes de regresión:

Parab:

Ho: $b=0$

$\mathrm{H} 1: \mathrm{b} \neq 0$

El valor $\mathrm{t}=-12,221$ tiene un valor $\mathrm{p}$-valor $=0,000<0,05$ por lo que rechazamos la hipótesis nula, lo cual nos indica que $(\mathrm{b} \neq 0)$.

\section{Para a:}

Ho: $a=0$

$\mathrm{H} 1: \mathrm{a} \neq 0$

El valor $\mathrm{t}=24,121$ tiene un valor $\mathrm{p}$-valor $=0,000<0,05$ por lo que rechazamos la hipótesis nula, lo cual indica que $(a \neq 0)$. 
Cuadro 11

COEFICIENTE DE DETERMINACIÓN Y ERROR ESTÁNDAR DE ESTIMACIÓN DEL MODELO DE REGRESIÓN INVERSO ESTIMADO

\begin{tabular}{|c|c|c|}
\hline$R$ & R cuadrado & Error estándar de la estimación \\
\hline 0,962 & 0,926 & 13797,108 \\
\hline
\end{tabular}

Cuadro 12

ANOVA DEL MODELO DE REGRESIÓN INVERSO

\begin{tabular}{|l|c|c|c|c|c|}
\hline & Suma de cuadrados & $g l$ & Media cuadrática & F & Sig. \\
\hline Regresión & 28428522006,134 & 1 & 28428522006,134 & 149,341 & 0,000 \\
\hline Residual & 2284322228,724 & 12 & 190360185,727 & & \\
\hline Total & 30712844234,857 & 13 & & & \\
\hline
\end{tabular}

La variable independiente es PBI.

\section{Contraste de regresión:}

Ho: $b=0$ (El modelo de regresión inverso no es significativo).

$\mathrm{H} 1: \mathrm{b} \neq 0$ (El modelo de regresión inverso es significativo).

El valor $F=149,131$ tiene un valor $p$-valor $=0,000<0,05$ por lo que rechazamos la hipótesis nula, entonces $b \neq 0$; lo cual nos indica que el modelo de regresión inverso es significativo. 


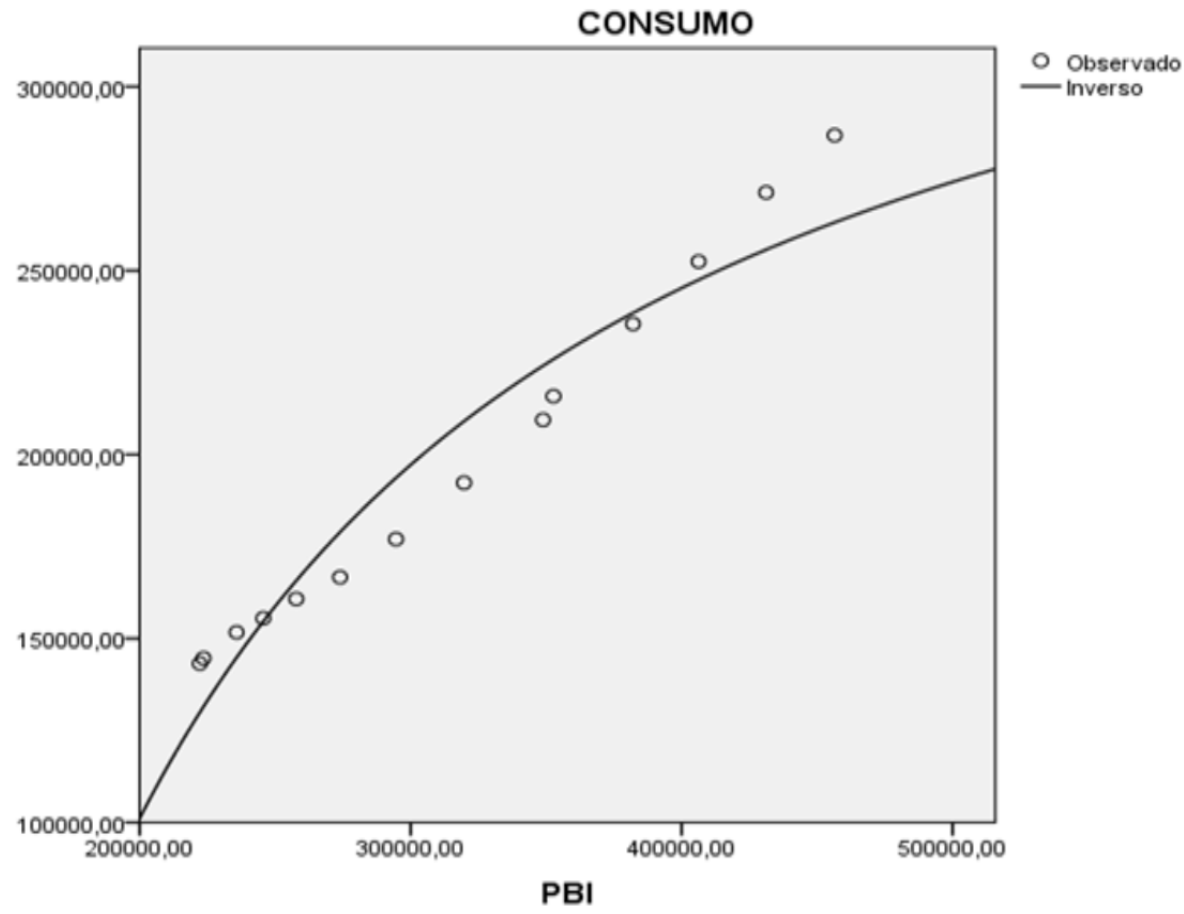

Gráfico 5. Curva del modelo de regresión inverso estimado.

Modelo de regresión inverso estimado:

$$
y=389446,419-\frac{5,767 * 10^{10}}{x}
$$




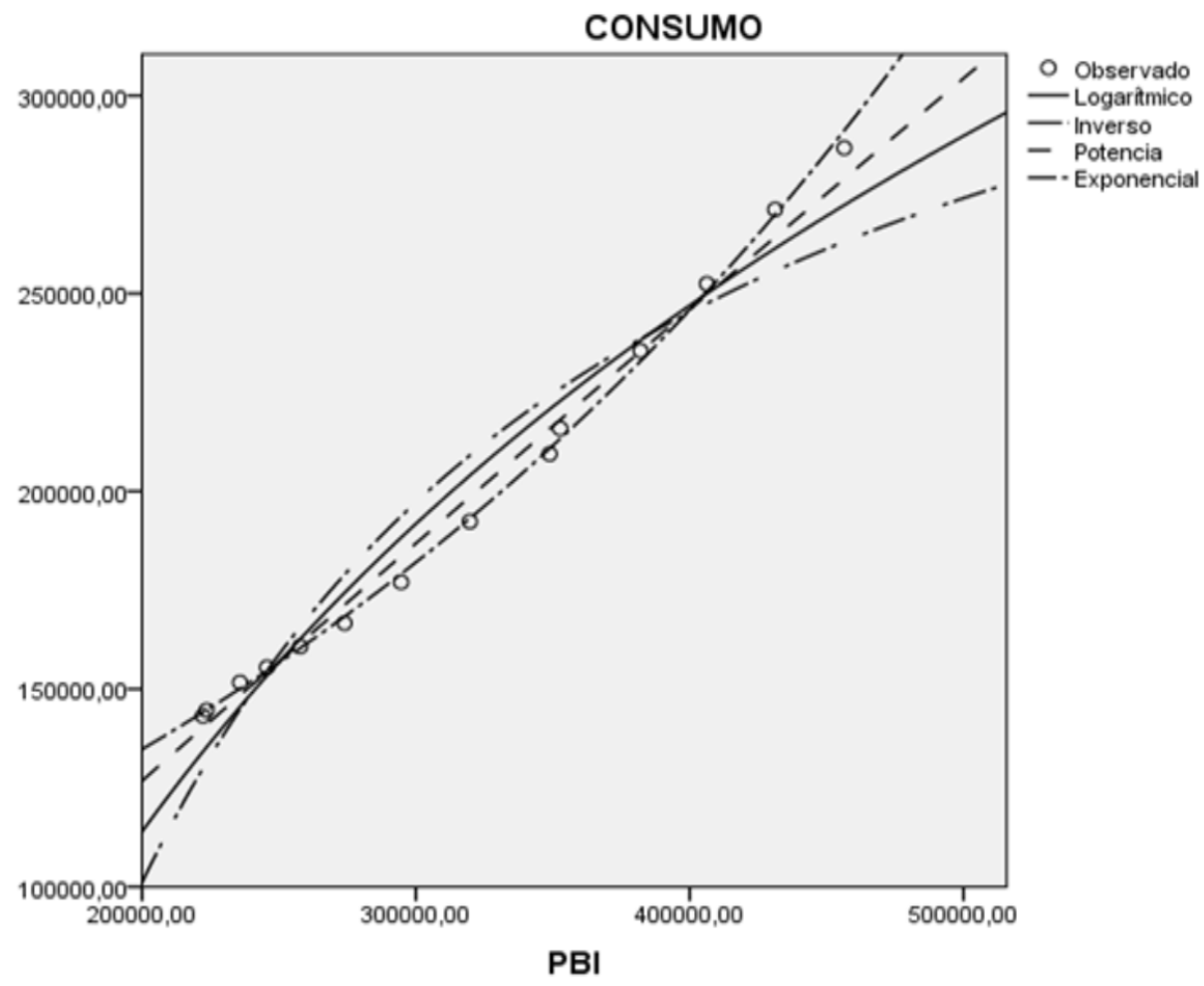

Gráfico 6. Curvas de regresión curvilínea sobre los datos observados.

Pronósticos para los años 2013, 2014 y 2016 de acuerdo al modelo de regresión exponencial estimado:

Año 2014: $\hat{y}=300801,11$ millones de soles.

Año 2015: $\hat{y}=314842,92$ millones de soles.

Año 2016: $\hat{y}=333393,36$ millones de soles.

\section{DISCUSIÓN}

El Gráfico 1, muestra el diagrama de dispersión de los datos originales observados y se puede observar que tienen una tendencia no lineal o dicho de otro modo tendencia curvilínea. 
De los cuatro modelos de regresión curvilíneos propuestos y desarrollados se ha elegido el modelo de regresión exponencial por tener el menor error de estimación $(0,009)$ y el mayor coeficiente de determinación $(0,999)$ tal como se indica en el Cuadro 2.

El Gráfico 2, muestra el modelo de regresión exponencial estimado sobre los valores observados el cual guarda relación con el coeficiente de determinación el cual indica que el 99.9\% de la variabilidad del Consumo Final Privado está explicado por el Producto Bruto Interno Nacional (PBI).

El Gráfico 3, se observa que el modelo de regresión exponencial estimado tiene un mejor ajuste con los datos originales respecto a los otros modelos de regresión curvilínea: potencial, logarítmico e inverso.

El Cuadro 1, indica que la constante "a" y el coeficiente de regresión "b" según el modelo de regresión exponencial estimado son significativos dado que los $\mathrm{p}$-valor $=0,000$ es menor que 0,05 .

El Cuadro 3, la prueba ANOVA nos indica que el modelo exponencial estimado es significativo ya que $\mathrm{p}$-valor $=0,000$ es menor que 0,05 .

Se descartan los modelos de regresión potencial, logarítmico e inverso por tener error estándar de estimación más altos, a pesar que el término constante y el coeficiente de regresión son significativos, además del ANOVA de cada modelo que indica que el Producto Bruto Interno Nacional (PBI) influye sobre el Consumo Final Privado.

En el Gráfico 6, se aprecia las curvas de regresión estimadas de los modelos de regresión en estudio, en el que se observa que el modelo de regresión exponencial tiene un mejor ajuste sobre los datos observados.

\section{CONCLUSIONES}

- El coeficiente de determinación $r^{2}=0,999$ indica que los datos se ajustan al modelo de regresión exponencial estimado.

- El Consumo Final Privado depende exponencialmente del Producto Bruto Interno Nacional (PBI).

- Frente al modelo de regresión potencial, logarítmico e inverso se eligió el modelo exponencial por tener el menor error de estándar de estimación. 
- La diferencia del error estándar de estimación entre el modelo exponencial y potencial es mínima. El modelo potencial puede superar al modelo exponencial para un mayor número de observaciones.

- Los pronósticos para el Consumo Final Privado para los años 2014, 2015, y 2016 son de 300801,11, 314842,92 y 333393,36 millones de soles respectivamente.

\section{REFERENCIAS BIBLIOGRÁFICAS}

De la Fuente, D., Pino, R. \& Parreño, J. (1995). Métodos cuantitativos de previsión. España: Servicios de Publicaciones de la Universidad de Oviedo.

Garcia, J.A., Gonzales, C. \& Ruiz, G. (2016). Estadística Empresarial (4 ed.). España: Servicio de Publicaciones de la Universidad de Cádiz.

Hernández, R., Fernández, C., \& Baptista, P. (2006). Metodología de la investigación. Obtenido de https://ebookcentral.proquest.com/lib/bibliocauladechsp/detail.action?docID $=4721683$

Instituto Nacional de Estadística. (20 de noviembre de 2015). Nota de prensa. Obtenido de https:/www.inei.gob.pe/prensa/noticias/consumo-final-privado-crece-33-8739/

Instituto Nacional de Estadística. (21 de noviembre de 2017). Nota de prensa. Obtenido de http://m.inei.gob.pe/prensa/noticias/pbi-crece-25-en-tercer-trimestre-impulsado-por-lademanda-interna-y-externa-10196/

Instituto Nacional de Estadística. (22 de agosto de 2016). Nota de prensa. Obtenido de https://www.inei.gob.pe/prensa/noticias/consumo-final-publico-y-privado-contribuyeroncon-el-crecimiento-del-producto-bruto-interno-del-segundo-trimestre-de-2016-9259/

Marulanda, C. (02 de abril de 2016). Análisis del PIB per cápita y del consumo privado en España: gráficos, Filtro de Hodrick-Prescott, ciclo y análisis con R. Obtenido de http://finanzaszone.com/analisis-del-pib-per-capita-y-del-consumo-privado-en-espanagraficos-filtro-de-hodrick-prescott-ciclo-y-analisis-con-r/

Pedraza, O. (2014). Modelo de plan de negocios: Para micro y pequeña empresa. México: Grupo editorial patria.

Rodríguez, J. (2006). Análisis de regresión lineal simple del consumo privado en ámerica latina. Obtenido de https://www.academia.edu/23875811/An\%C3\%A1lisis_de_regresi\%C3\% B3n_simple_de_consumo_privado_en_Am\%C3\%A9rica_LatinaFINAL_1_

Webster, A.L. (2001). Estadística aplicada a los negocios y economía (3ra. ed.). Colombia: Interamericana S.A. 